\title{
Recuperación de conocimientos tradicionales de gestión del paisaje de las huertas valencianas, propuestas de aplicación
}

\author{
Xavi Delgado Franco y Carles Rodrigo Alfonso \\ Pebrella ATF Consultora S.L. \\ xavier@pebrellaconsultora.com, carles@pebrellaconsultora.com
}

\begin{abstract}
Resumen. En los últimos años se han desarrollado diversas iniciativas de recuperación de saberes tradicionales ligados a la gestión agraria del territorio que desarrollaban los habitantes de las diferentes huertas valencianas. De esta forma se ha conseguido catalogar un interesante corpus teórico, sobre todo vinculado a actividades agrarias, que sin embargo está teniendo una aplicación práctica muy limitada pese al elevado interés que presenta. Más si cabe, en un ámbito territorial necesitado de nuevas propuestas de sostenibilidad que garanticen tanto su mantenimiento como viabilidad futura. Más allá de la aplicación dentro de la parcela agraria o del propio hogar estos conocimientos pueden ser útiles e incluso ser la base de nueva actividad en ámbitos variados.
\end{abstract}

Palabras clave: Huerta, paisaje, gestión, saber tradicional.

\begin{abstract}
There have been various initiatives recently dealing with the recovery of traditional knowledge related to agricultural land management which was developed by the inhabitants of different Valencian "Huertas". This has created an interesting theoretical corpus, mainly linked to agricultural activities, which however is having a very limited practical application despite its high interest. An increasing concern due to the need of new proposals in order to ensure both sustainability and future viability of these areas. This knowledge can be useful and become the basis of new activity in different fields beyond the application within land plot or home.
\end{abstract}

Keywords: Irrigation, landscape, management, traditional knowledge.

\section{Introducción}

En los últimos años el paisaje de las huertas valencianas ha experimentado notables cambios vinculados a la variación del uso y percepción de estos paisajes culturales y, sobre todo, a la evolución de la relación entre el paisaje cultural de las huertas y las ciudades en torno a las que se articula. Se podría afirmar que la década de los 90 del siglo pasado supuso un punto de inflexión en esta relación. En esos años, mientras la rentabilidad de la agricultura especializada de las huertas periurbanas valencianas disminuía dada la imposibilidad de competir con los precios de países terceros, se comenzaba también a poner en cuestión las implicaciones ambientales del modelo productivista que se había adoptado en las últimas décadas.

Casualmente o no, este cuestionamiento coincidió con el inicio de una de las épocas de crecimiento urbanístico más desatado del área metropolitana de las medianas y grandes ciudades en torno a las que se articulan históricamente estas huertas (València, Castelló, Alacant, Elx, Alzira, Xàtiva, Sagunt, etc.); de forma que se aceleró una feroz competición entre diferentes usos y actividades por ocupar los huecos que iba dejando la huerta en recesión. No se trataba de una dinámica nueva ya que el paisaje de las huertas periurbanas había tenido que hacer frente a otros periodos de crecimiento urbano y a la extensión de monocultivos como el de los cítricos que habían alterado ya notablemente su fisonomía tradicional. La introducción de nuevas técnicas agronómicas como el riego localizado o los invernaderos fueron también hitos de un cambio paisajístico y cultural de gran calado. 
Sin embargo, por primera vez, al menos de forma articulada; se comenzó a cuestionar si estos cambios eran positivos para las huertas y las propias ciudades. En ese contexto se plantearon diversas iniciativas encaminadas a defender el papel ambiental y cultural de estos espacios y su relación simbiótica con las ciudades que circundan y a reconocer el importante valor que tenían los conocimientos, prácticas y saberes de los diferentes gestores que administraban tradicionalmente este paisaje.

Ya en el siglo XXI estos movimientos ciudadanos, como por ejemplo Per l'Horta, están plenamente consolidados y desarrollan más que notables iniciativas de estudio y recuperación del paisaje de las huertas periurbanas. Sin embargo, dado el escaso apoyo institucional que reciben estos colectivos, estas iniciativas no suelen ir más allá de la recuperación o mantenimiento de la actividad agraria en algunas parcelas. Un trabajo necesario y que supera con creces los resultados que cabría esperar de una situación marcada por la falta de apoyo administrativo, pero que debe verse complementado con nuevas propuestas que permitan valorizar las huertas mediante nuevas rentabilidades económicas, sociales o culturales y en el que el corpus teórico desarrollado por sus gestores tradicionales debe de tener un papel primordial. No puede existir nada tradicional sin una tradición en que basarse, es decir; no puede haber un paisaje tradicional sin un conocimiento tradicional que sustente su mantenimiento.

Desde la perspectiva de los autores, técnicos en desarrollo local y territorio, se percibe el paisaje cultural de las huertas y el conocimiento tradicional ligado a su gestión como un espacio con un notable potencial para desarrollar múltiples actividades alternativas que justifiquen no solo su mantenimiento sino también su recuperación allá donde se haya perdido. La presente comunicación analizará posibles vías de valorización de estos espacios mediante el aprovechamiento de estos conocimientos de forma que se puedan mantener tanto el paisaje cultural como el corpus teórico que lo sustenta.

\section{La valorización del paisaje. El valor (los valores) de las huertas valencianas y el valor de su gestión.}

No es el objetivo de esta comunicación establecer ni cuáles son estos valores ni su grado de interés, dado que eso requeriría de un análisis y exposición más largo que el que aquí se pueda presentar. En cualquier caso haremos un breve repaso que ayude a contextualizar la presente comunicación.

El paisaje tiene un alto valor per se, ya que se puede definir como la expresión física de la relación entre ser humano, cultura y territorio. Autores como Tello (1999) han expuesto ya la importancia de esta relación y la propia constitución española en sus artículos 45 y 46, dedicados a la conservación del medio ambiente y del patrimonio, reconoce la obligación del estado de velar por su mantenimiento y mejora. De este reconocimiento constitucional se deriva un corpus legislativo estatal y autonómico al que se sobrepone otro de carácter europeo que debería de ser suficiente para garantizar la preservación de estos valores. Sin embargo, el paisaje/territorio es también percibido por algunos como un bien de consumo o como un mero soporte de actividades por parte de otros. Interpretaciones que nos han llevado al marco actual en el que la legislación protectora del paisaje se contempla desde parte de la administración y muchos ámbitos privados como una molestia que se debe a restringir a ámbitos muy concretos. 
Se hace así necesario reconocer el valor añadido de los paisajes culturales de las huertas valencianas si se quiere crear un estado de opinión favorable a su conservación. Establecer el valor de un paisaje siempre es complejo y más si se pretende hacerlo desde criterios objetivos. Autores como Marangón y Tempesta (2009) afirman que el valor del paisaje deriva principalmente de las funciones que desarrolla, es decir de las necesidades que satisface y por tanto de los beneficios que produce. Otros autores como Luginbühl (2008) consideran que estos valores se deben extender más allá y abarcar ámbitos más subjetivos si cabe al relacionar el paisaje con el bienestar tanto individual como social. Una relación que lleva a este autor a afirmar: se debe, por tanto, ir más allá. Sobre todo explorar los esfuerzos de la sociedad por ordenar el paisaje, con la finalidad de satisfacer mejor las necesidades esenciales, pero también de responder a aquellos anbelos que tienen menos relación con la naturaleza física (Luginbühl, 2008). Unos valores que ha llevado a otros autores a relacionar la calidad del paisaje con la calidad de vida y la salud de aquellos que lo viven y contemplan (Pol et al., 2008).

Por otra parte cabe considerar que la preservación del paisaje cultural de las huertas valencianas debe de ir más allá de crear un reservorio estético de esencias tradicionales que se defiende desde algunos ámbitos sociales y administrativos. Conservar unas parcelas de huerta y algunos edificios emblemáticos puede tener su interés pero es una tarea más paleontológica que de dinamización social o económica; que es lo que la auténtica preservación del paisaje requiere. La pervivencia de este paisaje cultural y de los valores que puede ofrecer debe de vincularse necesariamente al interés de su uso y mantenimiento. Unas rentabilidades que van más allá de lo económico y social sin que ello implique necesariamente descuidar estos ámbitos que probablemente sean los de más fácil valorización.

La pervivencia del paisaje de nuestras huertas no se puede desligar de la pervivencia del modelo de gestión que las sustenta y otorga buena parte de sus valores añadidos. Un modelo que sin caer en esencialismos debe de adaptarse y evolucionar con los tiempos, pero que si es abandonado y pierde sus principales líneas vertebrales nos lleva a un remedo de huerta vacío de contenido. Espacios que parecen pero no son y que camuflan bajo una apariencia de sostenibilidad, o al menos inocuidad, unas dinámicas negativas para sociedad y territorio. La buerta como espacio cultural no puede desligarse del modelo de gestión que le dio luz. No se puede tener un espacio tradicional sin la tradición que lo vertebra. Un modelo basado en principios como el valor del consenso, el papel del colectivo y del individuo y que aprovechaba soluciones de bajo coste y baja intensidad ambiental para resolver problemas complejos como los derivados de la erosión, la pérdida de fertilidad, la falta y el reparto de caudales hídricos, la resolución de conflictos o la gestión de espacios e infraestructuras comunes.

Este modelo de gestión se basaba en un corpus teórico de carácter empírico que se transmitía a sus usuarios de múltiples formas y que permitía una gestión muy eficaz, aunque con frecuencia de carácter inconsciente por lo que se refiere tanto a la mecánica de la práctica o gestión como del conjunto de beneficios que comportaba. Actualmente contamos con la ventaja de poder analizar estas prácticas y poder actuar de forma consciente potenciando aún más sus aspectos positivos.

Se podría aducir que la escala de los problemas ha variado por la intensificación de actividades y de usos y ocupación del paisaje, aunque cabe considerar que también ha variado nuestra capacidad de organización compleja y la capacidad tecnológica; de forma que partiendo de la filosofía del conocimiento tradicional se puede articular una respuesta más eficaz. Cabe también tener en cuenta que estos conocimientos tienen un alto valor como elemento cultural pero puede que no más allá. El paisaje es un elemento vivo y en evolución y, por tanto, parte de este conocimiento es obsoleto desde el momento en que su marco 
referencial ha desaparecido. También se han producido cambios tecnológicos y sociales que han permitido articular mejores respuestas a determinados problemas de gestión del paisaje que las que se desarrollaban tradicionalmente. No todo el conocimiento tradicional va a tener una aplicación directa en la gestión actual del paisaje, pero todo el conocimiento tradicional de gestión del paisaje tiene un valor culturaly social y por tanto debe de ser preservado.

3 Valorización de los conocimientos tradicionales de gestión del paisaje de las huertas valencianas.

Creemos que esta propuesta de valorización debe de abordarse desde una perspectiva doble, por un lado abordar la cuestión metodológica de cómo recuperar estos conocimientos y por el otro analizar como valorizar los conocimientos recuperados y adquiridos. Nos extenderemos más en el segundo ámbito dado que el primero parte de metodologías de investigación bastante comunes aunque desde perspectivas y enfoques novedosos.

\section{1 ¿Cómo recuperar los conocimientos tradicionales?}

La recuperación de los conocimientos tradicionales vinculados a la gestión del territorio/paisaje se ha abordado tradicionalmente desde la perspectiva de la etnología/antropología y a partir de la metodología típica de investigación documental + trabajo de campo (entrevistas registradas por diferentes medios y/o visitas de estudio a espacios concretos) + análisis e interpretación de los resultados.

En las últimas décadas este enfoque tradicional se ha visto complementado por al menos otros dos que han permitido ir más allá del estudio científico y centrar el objetivo en la concesión de resultados prácticos: Los ecosaberes y los UrbanLabs.

\subsubsection{La recuperación de ecosaberes.}

Los ecosaberes se articulan en torno a la relación compleja que existe entre un territorio, sus recursos naturales y las personas que lo habitan; prestando especial atención a la capacidad que tienen ciertas sociedades tradicionales de tener en cuenta las particularidades ecológicas locales, gestionar la complejidad (agro-forestal, territorios concéntricos...) y organizarse (acceso a los recursos, gestión de los conflictos...). El objetivo de la recopilación de ecosaberes es encontrar respuestas en estos conocimientos a los grandes retos ambientales a los que se enfrentan las sociedades actuales de forma que se puedan orquestar respuestas desde una perspectiva sostenible.

Los autores de esta comunicación participan desde más de una década en un equipo internacional de investigadores y divulgadores que se han marcado a largo plazo el objetivo de crear una enciclopedia que recoja estos ecosaberes a escala mundial (Ecologie Paysanne, 2014).

\subsubsection{Los UrbanLabs.}

Otro ámbito de estudio/aplicación que ha surgido en los últimos años de este corpus empírico de gestión tradicional del territorio es el de los UrbanLabs. Estos foros de reflexión y debate en los que se aborda el análisis del propio territorio desde múltiples enfoques y colectivos con la finalidad de ofrecer soluciones de futuro, suelen dedicar_parte 
de sus actividades al estudio de la relación entre la ciudad y los espacios periurbanos que la rodean. Por el momento, las iniciativas a este nivel que se están desarrollando en territorio valenciano están en una fase muy inicial, aunque en territorios vecinos como Catalunya ya se han cosechado resultados notables.

En los UrbanLabs este conocimiento tradicional se valoriza en sentidos muy diversos y mediante vías abiertas. Primariamente el conocimiento tradicional funciona como una herramienta de comunicación intergeneracional, intergrupal e intercultural al intercambiarse saberes y experiencias entre los diferentes participantes. A partir de ahí, y una vez incorporado al acervo colectivo el conocimiento tradicional ligado a la gestión del territorio, se valoriza, aprovecha y difunde tanto el saber cómo las posibilidades que ofrece.

\subsection{Valorización de conocimiento tradicional sobre la gestión del paisaje de las huertas valencianas.}

No se puede iniciar este apartado sin hacer una consideración inicial que creemos de justicia. Aunque pueda sonar contradictorio, la gestión tradicional del paisaje de la huerta es, a ojos del investigador, extraordinariamente moderna. Se trata de una modernidad de carácter conceptual ya que la gestión tradicional del paisaje de la huerta encajaba completamente en el concepto de sostenibilidad. Esta gestión permitía el uso continuado del territorio sin causar agotamientos ni daños que comprometiesen la continuidad de la explotación o de la propia sociedad. Una gestión todavía más meritoria si se tiene en cuenta que, contrariamente a la que se desarrollaba en otros paisajes culturales tradicionales, se trataba de una gestión de carácter intensivo; en simbiosis con áreas urbanas de cierta importancia y con una orientación comercial de parte de sus producciones al menos desde tiempos bajomedievales (Piqueras, 1985).

El marco conceptual de la gestión tradicional del paisaje de las huertas valencianas encajaría perfectamente con propuestas y modelos de desarrollo social y económico de última generación como la economía circular que se propugna desde la Ellen MacArthur Foundation (2014) o del Cradle to Cradle que defienden Braungart y McDonough (2005). La sostenibilidad de procesos y resultados está plenamente integrada en el $A D N$ de la gestión tradicional de estos paisajes. La baja intensidad ambiental de las actuaciones, la multiplicidad de los valores añadidos de cada proceso y producto, la inexistencia práctica de residuos, la integración y cooperación de las actividades o el enfoque integral de problemáticas y soluciones son solo algunas muestras de esta afirmación.

\section{3 Ámbitos concretos de aplicación de los conocimientos tradicionales de gestión del paisaje cultural de las huertas valencianas.}

El abanico de conocimientos tradicionales ligados a la gestión del territorio es tan amplio y afecta a tan diversos ámbitos que sistematizarlos íntegramente tanto en categorías como en posibilidades de aprovechamiento excede con mucho la capacidad de la presente comunicación. Por ello, nuestro análisis se ceñirá a marcar un esbozo de categorización y posibilidades más que a una descripción exhaustiva. En esta voluntad de simplificar hemos optado por una clasificación orientada a ámbitos de aplicación en lugar de otra, que tendría sentido en un ámbito de estudio más amplio, basada en la propia tipología de los conocimientos. 


\subsection{1 Ámbitos de aplicación inmediata en gestión agraria, gestión territorial y}

entorno doméstico. Existe un amplio catálogo de conocimientos de carácter tradicional que tiene una aplicación sencilla e inmediata y que se pueden traducir en un aumento de la sostenibilidad en determinados espacios y ámbitos. Por ejemplo, saberes y conocimientos ligados a la conservación tradicional de alimentos y su procesado pueden ayudar a disminuir el consumo energético al reducirse la necesidad de empleo de cadena del frío para conservación. La cadena de valor añadido de esta conserva va mucho más allá de este beneficio inicial. Su elaboración implica también que se puede consumir el producto fuera de temporada sin recurrir a productos de invernadero o traídos de orígenes lejanos de forma que se reduce la huella de carbono y se reduce el riesgo de consumir productos con trazas más que importantes de agroquímicos. De este modo se superpone a todo ello el valor cultural de la propia práctica y la posibilidad añadida de contribuir al mantenimiento de variedades tradicionales menos aptas para el comercio internacional aunque mucho más adaptadas a las condiciones locales.

El empleo de variedades y razas tradicionales en explotaciones amplía todavía más allá esta cadena de valor añadido. No es necesario extenderse en la necesidad e interés de mantener el patrimonio genético. Un patrimonio que se pierde a marchas aceleradas y que nunca se sabe cuándo puede ser necesario en el futuro. A este interés cabe añadir que la adaptación a las condiciones locales de estas variedades y razas implica una mayor resistencia a las condiciones extremas que se den en la zona y una menor dependencia de insumos con riesgo potencial como agroquímicos o determinados medicamentos ganaderos. Las cualidades organolépticas de estas variedades y razas locales suelen ser también mejores que las de aquellas creadas con orientación al mercado habitualmente seleccionadas por criterios como la productividad o la resistencia a viajes prolongados. Así mismo, estas variedades y razas tradicionales fueron seleccionadas en su momento no sólo por su adaptación al medio sino también por su mejor encaje en la gastronomía local; de forma que su mantenimiento y empleo añade a beneficios ambientales y culturales los económicos y sociales de poder mejorarse la oferta gastronómica local.

Obviamente el conocimiento tradicional agrario tiene un papel primordial en este ámbito. En un panorama creciente de interés por la agricultura y en el que proliferan experiencias como huertos urbanos, huertos colectivos o formas de agricultura alternativas el corpus teórico desarrollado por los gestores tradicionales del territorio tiene un amplio abanico de aplicación que va desde el control de plagas, el control de la erosión, la fertilización, la administración de caudales hídricos hasta el desarrollo de técnicas para combatir los daños por eventos climáticos extremos. Constituye un caudal de conocimiento que puede contribuir a mejorar mucho la relación entre sociedad y territorio y también a mejorar notablemente qué se consume y cómo se consume en nuestras sociedades. Es también destacable el papel que puede desempeñar este conocimiento en unos mercados competitivos en el que la diferenciación del propio producto es la clave para aquellos que no pueden o no aspiran a competir mediante precios bajos.

Estos conocimientos pueden aplicarse también de forma inmediata a la resolución en ámbitos territoriales de tamaño medio o gran tamaño. La aplicación de determinadas técnicas o conocimientos puede suponer una mejora destacada de algunas problemáticas. Un buen ejemplo es del aprovechamiento de recursos silvestres que tenían un papel ecológico importante y la recuperación de parte de estos conocimientos que ayudaría a solventar problemáticas como el control de plagas, el mantenimiento de la biodiversidad o la dependencia de recursos foráneos. Un buen caso demostrativo es el empleo de la caña común (arundo donax). En nuestra sociedad tradicional tuvo un empleo y uso intensivos 
siendo un elemento casi tan omnipresente como los actuales plásticos. Se empleaba en ámbitos tan diferentes como la propia construcción de las viviendas (canyís), la elaboración de objetos de uso cotidiano (instrumentos musicales, juguetes, aventadores, etc.), la fabricación de herramientas, la construcción de estructuras efímeras de uso agrario y uso variado, etc. Al tratarse de una planta foránea con una increíble capacidad de propagación el abandono del uso de la planta se ha traducido sobre el terreno en su reconversión en planta invasora de cauces que causa problemas graves como el aumento del riesgo de inundación e incendio, la pérdida de biodiversidad, el cierre del paisaje, etc. Se están empleando diversas técnicas para intentar reducir este impacto negativo, pero a la desventaja de su elevado coste añaden que la caña destruida se convierte en un residuo sin uso.

La gran mayoría de los estudios coinciden en que la lucha contra la caña como especie invasora debe de ser continuada ya que si no en muy poco tiempo se pierde cualquier efecto positivo que se haya generado. De hecho la caña no era un problema en la sociedad tradicional porque se explotaba tanto que estaba muy acotada y casi en riesgo de recesión, dándose casos de enfrentamientos más o menos serios por el derecho de explotación de la planta allá donde escaseaba más. La recuperación de saberes tradicionales en este ámbito puede ser una buena estrategia complementaria a otras (como por ejemplo aprovechamientos energéticos o papeleros) que permita establecer de nuevo un uso intensivo de esta planta invasora que la mantenga bajo control. En este sentido ya se están desarrollando interesantes iniciativas en territorio valenciano y en otros lugares vinculadas a su empleo como material constructivo de bajo coste, sostenible y renovable (Econstrucció, 2014). De nuevo los beneficios van más allá del ahorro que pueda suponer su empleo o de los generados por mantener bajo control la planta, puesto que se deben tener en cuenta también otros elementos que se reducen como el empleo de determinados materiales de construcción poco sostenibles o la reducción de la cadena de transporte y toda su problemática derivada.

\subsubsection{La gestión del paisaje tradicional de las huertas como elemento patrimonial y} como escenario cultural. Como ya se ha expuesto anteriormente, los autores, estamos por la preservación de un paisaje cultural de las huertas valencianas dinámico y vivo más que por la conservación de algunos rincones fósiles que funcionen más como museo de tradiciones que como hábitat social. Pese a ello, no se puede soslayar ni dejar de lado que un paisaje cultural de huerta es un elemento patrimonial de primer nivel y de elevada singularidad. Representa un patrimonio susceptible de ser aprovechado didáctica, cultural y turísticamente. Un paisaje que a su propio valor añadido patrimonial suma el de su valor como escenario singular para cualquier tipo de actividad en relación directa o no con él. El turismo y las industrias de la cultura pueden aprovechar los paisajes de huerta como objeto comercial pero también como escenario interesante. Se puede desarrollar un itinerario didáctico o turístico por la huerta o simplemente aprovechar la huerta como escenario como un paseo, aunque en los tres casos se estará valorizando este paisaje cultural.

Un aprovechamiento que se puede dar tanto desde ámbitos públicos como privados y con finalidades lucrativas o no. Un buen ejemplo de valorización de esta gestión es el que se ha desarrollado con el Web Paisatges Culturals a la Reial Séquia de Montcada (2014) que combina la investigación con la didáctica y la divulgación o las diferentes rutas turísticas que se han desarrollado alrededor de la huerta de la ciudad de València y sus producciones. 
3.3.3 La gestión del paisaje tradicional de las huertas como espacio social y territorial compartido. La gestión del paisaje tiene un fuerte componente social. Junto al conjunto de técnicas y prácticas de cualquier tipo se encuentra un corpus normativo elaborado en torno a consensos generales que garantizan su respeto por todos los estamentos y afectados. Este corpus normativo es por lo general de carácter consuetudinario aunque en ocasiones, como por ejemplo muchas regulaciones de acequias de riego, también tendrá una expresión escrita.

Este conjunto de regulaciones abarca prácticamente todos los ámbitos con ejemplos que van desde el orden en que se lavan diferentes elementos en un lavadero (ropa, ajuar doméstico, personas...) a cuestiones complejas como el mantenimiento de infraestructuras hidráulicas o camineras que suman centenares de kilómetros de longitud y un amplio catálogo de formas. Una complejidad dado que afectaba tanto a espacios públicos como privados creando a veces situaciones mixtas en las que el acceso a un bien privado podía convertirse en público. Representan un buen ejemplo de lo que se afirma las regulaciones que establecían cuándo y en qué condiciones ganados ajenos podían acceder a un campo (por ejemplo en determinadas épocas de barbecho o bajo otras circunstancias excepcionales).

Por otro lado, existe una tercera vertiente de alto interés en el estudio y aprovechamiento de estos conocimientos; la relacionada con la organización de los trabajos comunales y la resolución de conflictos. Existen metodologías tradicionales con pautas reconocibles que permiten el desarrollo de determinados trabajos conjuntos como las dulas de diversos animales (cerdos, cabras, equinos, etc.) con pastores rotativos o fijos, los intercambios de trabajo (el tornallom), los sistemas colectivos de crédito, o el mantenimiento conjunto de espacios bien mediante agrupaciones de trabajadores bien mediante responsabilidades individuales sobre secciones concretas del espacio conjunto. Por lo que se refiere a la resolución de conflictos, cabe señalar que existen gran cantidad de mecanismos aparte de los conocidos tribunales de aguas, como por ejemplo los dedicados a resolver los conflictos resultantes de los daños causados por pastores o los sistemas que velaban por el mantenimiento de lindes.

En un entorno complejo como el actual en el que la sociedad civil demanda una mayor participación, algunas administraciones retroceden en sus responsabilidades y las sociedades se componen de múltiples colectivos con origen e intereses muy diferentes, toda esta mecánica de resolución de conflicto y de pautas de cooperación con un fin común tiene un amplio campo de desarrollo. Los UrbanLabs parecen un escenario perfecto para esta aplicación que de forma informal ya se está aplicando el seno de algunos colectivos que han recuperado algunas formas de trabajo agrupado.

3.3.4 La gestión del paisaje tradicional de las huertas como elemento de sostenibilidad a largo plazo: Ciclos integrales e integrados. La gestión del paisaje tradicional se caracterizaba, como ya hemos expuesto en párrafos anteriores por su alto grado de sostenibilidad a largo plazo; marcada por el empleo sostenido de recursos locales de carácter renovable. Esta sostenibilidad se articulaba en una serie de ciclos integrales y corta distancia de aprovechamiento, abastecimiento y consumo que permitían el aprovechamiento con escasas pérdidas. Estos ciclos integrales también estaban integrados, es decir se entrelazaban de forma que se transferían elementos creando flujos y dinámicas positivas tanto de gestión social como de gestión territorial. 
Un buen ejemplo de lo que acabamos de expresar es el de la gestión de los residuos. Un problema creciente en sociedades avanzadas como la nuestra y que en el caso valenciano se halla en un punto crítico. La sociedad tradicional de nuestras huertas era una sociedad de residuo cero. Los objetos se reparaban, reutilizaban y al final de su vida útil eran reconvertidos en otros objetos o reciclados; existiendo redes de profesionales (quincalleros, cocoueros/recoveros, traperos, etc.) encargados de su recogida y tratamiento. La materia orgánica se destinaba tanto al consumo de animales domésticos como, sobre todo, a unos campos siempre necesitados de fertilizantes. El Cradle to Cradle de Braungart y Mc Donough se aplicaba en los paisajes culturales de nuestras huertas (y en muchos otros ámbitos) bastante antes de ser definido conceptualmente hace unos años. Este modelo de gestión de residuos reducía por una parte el empleo de materias primas y por tanto la presión sobre el territorio, pero tenía interrelaciones mucho más complejas: El uso agrario de los residuos orgánicos urbanos, que eran recogidos casa por casa por los agricultores en algunas épocas y posteriormente con la creación de la Sociedad de Agricultores de la Vega, mediante sistemas más complejos; permitía ahorrar en insumos agrarios y depender menos de agroquímicos. No se acababan aquí los beneficios de este ciclo integral, se suele soslayar que de esta forma y mediante este aprovechamiento los residuos se convertían en un elemento de cohesión social que relacionaba a los agricultores de l'Horta con la población urbana. La imbricación de estos dos ciclos, el de los residuos y el de las producciones agrarias, se convertía así en un factor clave en la simbiosis entre la ciudad y la huerta que la rodea; en uno más de los puntales de las relaciones sociales necesarias para la convivencia.

La recuperación de estos ciclos, de su filosofía de funcionamiento a partir de recursos y potencial endógeno, se pueden convertir en un importante motor de desarrollo local. Un modelo de gestión austera, muy en relación_con los tiempos que corren, aunque centrando la austeridad en la economía de los recursos no en otros elementos económicos cuyo ahorro siempre implica un gasto mayor a largo plazo. Un caso bastante ilustrativo sería el del ciclo integral del agua. Resulta sorprendente que cuanto más aumenta la tecnología de ahorro a nuestra disposición más disminuyen los caudales efectivos de los que se dispone. Se precisa una mirada tradicional para comprender que el ahorro que se consigue en un punto no se puede trasladar automáticamente a un incremento similar del consumo en otro; existen mil y un elementos e interrelaciones complejas que cabe comprender mejor. La sequía estructural que padecemos, ahora agravada por otra de carácter climático, depende más de factores de gestión que de factores tecnológicos. Sólo una gestión integral como la que se desarrollaba de forma tradicional puede garantizar a largo plazo la sostenibilidad del ciclo.

Seguir estos ciclos, cerrarlos y crear otros nuevos a partir del estudio de los tradicionales es un elemento clave a la hora de planificar el futuro no sólo de los paisajes culturales de las huertas sino del conjunto del territorio y sociedad que los sustenta. Experiencias como las que se están desarrollando en Catalunya con la recogida puerta a puerta (Associació, 2014) de los residuos separados en origen doméstico (más de 100 que agrupan a más de 300.000 habitantes) son una buena prueba de cómo la recuperación de un modelo de gestión tradicional aliado con la tecnología y posibilidades actuales permite convertir lo que inicialmente era un problema de gestión y ambiental en un motor del desarrollo económico y social local.

\section{Conclusiones}

En los últimos años se han impulsado diversas iniciativas de recuperación de saberes tradicionales ligados a la gestión agraria del territorio que desarrollaban los habitantes de las diferentes huertas valencianas. De esta forma se ha conseguido catalogar un interesante 
corpus teórico, sobre todo vinculado a actividades agrarias, que sin embargo está teniendo una aplicación práctica muy limitada pese al elevado interés que presenta. Más si cabe, esta escasa aplicabilidad se produce en un ámbito territorial necesitado de nuevas propuestas de sostenibilidad que garanticen tanto su mantenimiento como viabilidad futura.

A lo largo de nuestra comunicación hemos realizado una pequeña síntesis del estado de la cuestión, establecido el valor de los paisajes culturales tradicionales más allá de aspectos meramente culturales o folklóricos. En ella se ha esbozado una perspectiva sintética e integradora sobre las principales líneas de trabajo a desarrollar con la finalidad de que la gestión tradicional del paisaje se convierta en una herramienta para su mantenimiento por la vía de reforzar su papel patrimonial junto a su utilidad para favorecer la mejora social y económica. En este sentido, la preservación parcial de este paisaje puede anular los efectos positivos que la simbiosis espacio habitado/huertas ha generado a lo largo del tiempo. Únicamente la preservación del paisaje cultural en su conjunto, aunque partiendo siempre de su adaptabilidad y necesidad de adaptación a las transformaciones en curso, garantiza el mantenimiento de las dinámicas positivas que genera.

En referencia a las vías de aprovechamiento y valorización del conocimiento tradicional ligado a la gestión del territorio, hemos identificado cuatro grandes ámbitos de aplicación diferenciados:

- Ámbitos de aplicación inmediata en la gestión agraria, la gestión territorial y el entorno doméstico.

- La gestión del paisaje tradicional de las huertas como elemento patrimonial y como escenario cultural.

- El desarrollo de la gestión del paisaje tradicional de las huertas considerado como un espacio social y territorial compartido.

- La promoción de la gestión del paisaje tradicional de las huertas como un elemento de sostenibilidad a largo plazo: impulsión de los ciclos integrales e integrados.

Cada uno de ellos, pese a mostrar características propias y disponer de diferentes metodologías de valorización y aprovechamiento, pueden compartir siempre el fondo común de la revitalización del conocimiento tradicional. De este modo resulta factible dotarlo de nuevos usos que generen beneficios sociales, ambientales y territoriales.

\section{Referencias}

Associació de municipis catalans per la recollida porta a porta (2014): http://www.portaaporta.cat.

Ecologie Paysanne (2014): http:/ / ecologie-paysanne.org.

Econstrucció (2014): http://econstruccio.wordpress.com, aunque existen muchas otras iniciativas de notable interés similares.

Ellen MacArthur Foundation (2014): http:/ /www.ellenmacarthurfoundation.org/

Braungart, M. Mc Donough W. (2005): Cradle to Cradle (De la cuna a la cuna): Rediseñando la forma en que hacemos las cosas. Madrid, McGraw-Hill. 
Luginbühl, Y. (2008): "Paisatge i benestar individual i social", en Nogué, Joan; Puigbert, Laura; Bretcha, Gemma (eds.), Paisatge $i$ salut. Observatori del Paisatge de Catalunya; (Plecs de Paisatge; Reflexions; 1), pp 13-35.

Marangón, F. Tempesta, T. (2009): "La valoració econòmica del paisatge. Una proposta d'indicadors", en Nogué, J.; Puigbert, L.; Bretcha, G. (eds.) Indicadors de paisatge. Reptes $i$ perspectives. Observatori del Paisatge de Catalunya (Plecs de Paisatge; Eines; 1), pp 77-106.

Paisatges culturals a la Reial Séquia de Montcada (2014): http://www.paisatgesculturalsrsm.org.

Piqueras, J. (1985): La Agricultura valenciana de exportación y su formación histórica, Madrid, Instituto de Estudios Agrarios, Pesqueros y Alimentarios.

Pol, E. ; Castrechini, A. y Di Masso, A. (2008): Paisatge i qualitat de vida. en Nogué, Joan; Puigbert, Laura; Bretcha, Gemma (eds.), Paisatge i salut. Observatori del Paisatge de Catalunya; (Plecs de Paisatge; Reflexions; 1), pp 36-51.

Tello, E. (1999): "La formación histórica de los paisajes agrarios mediterráneos: una aproximación coevolutiva", Historia Agraria, 19:195-212. 